\title{
A Stable Backbone-Based on Demand Multipath Routing Protocol for Wireless Mobile Ad Hoc Networks
}

\author{
Sujata V. Mallapur \\ Godutai Engineering College for Women, Kalburgari, Karnataka, India \\ E-mail: sujatavmallapur@gmail.com \\ Siddarama R. Patil and Jayashree V. Agarkhed \\ Poojya Doddappa Appa College of Engineering, Gulbarga, Karnataka, India \\ E-mail: \{pdapatil, Jayashreeptl\}@yahoo.com
}

\begin{abstract}
In mobile ad hoc networks (MANETs), high node mobility leads to frequent link breaks and creates complexities in route discovery, this effect on the quality of service (QoS) and degrades the systems performance. So, providing a high quality communication using stable links among mobile nodes is a challenging issue in MANETs. Existing stability based routing algorithms initiate estimation of route stability metrics during the routing process, results in increased delay and overhead. In order to overcome these issues, in this paper, we construct a stable backbone-based multipath routing protocol (SBMRP). Initially, the nodes with high residual bandwidth, residual power, link quality and low mobility are designated as candidate nodes. Then multiple paths are established between source and destination through these candidate nodes, thus forming a routing backbone. If any candidate node in the path tend to fail due to lack of bandwidth, energy or link quality, alternate path through other candidate node is established before path breaks. Proposed routing scheme has been compared with other three existing protocols: AODV, AOMDV and RSQR. The protocol performance has been evaluated in terms of packet delivery ratio, normalized routing load, delay and packet drop ratio.
\end{abstract}

Index Terms-MANETs, Candidate nodes, Residual bandwidth, Residual energy, Link quality.

\section{INTRODUCTION}

The mobility of nodes in mobile ad hoc networks (MANETs) causes the change in network topology; frequent path breaks and increases the packet drops [1] [2]. Other than node mobility, link failure may also depend on other factors, such as energy of node, bandwidth available for node and link quality etc. If link failure takes place in the network, frequent route discovery attempts could congest the network and also knock out the battery power at critical nodes. Multimedia applications requires the packets to be delivered in-order with negligible variance in the inter-packet delay, frequent changes in the routes traversed by the packets could result in out-of-order packet delivery with high jitter [3]. When any link of a path breaks, this path needs to be either repaired by finding another link if any or replaced with a newly found path. The rerouting operation cost, the scarce radio resource and battery power rerouting delay may also affect QoS and degrade the network performance.

Most of the routing algorithms proposed for ad hoc networks are based on on-demand routing [4 - 8]. In these routing algorithms, when a route is broken, route recovery and maintenance procedures are initiated. These procedures consumes substantial amount of resources (in terms of wireless bandwidth, power, processing capacity at nodes) and introduce extra delay in the network. The existing protocols try to improve routing efficiency either by using mobility prediction technique and energy consumption or link quality or residual bandwidth of a node. None of them address the combination of mobility, residual energy, residual bandwidth and link quality parameters to select the reliable nodes. In most of the existing stability based routing protocol link quality and residual energy of each node is estimated during the routing process which results in increased delay and overhead.

The proposed work is motivated by observing the limitations of the existing stability based routing protocols. The main aim of this work is to propose a reliable routing protocol for MANET. The proposed method attempts to maximize the link stability for the transmission of packets. By considering these issues, proposed to construct a stable backbone-based multipath routing protocol (SBMRP) for MANETs. Initially, the nodes with high residual bandwidth, residual power, link quality and low mobility nodes are designated as candidate nodes. Then multiple paths are established between source and destination through these candidate nodes, thus forming a routing backbone. If any candidate node in the path tend to fail due to lack of bandwidth, residual energy or link quality, alternate path through another candidate node is established. By simulation results, we show that proposed technique minimizes the 
overhead, packet drop, energy and increases the packet delivery rate.

\section{A. Contribution of the work}

The proposed protocol scheme is designed by observing the limitations of existing on demand multipath routing protocol schemes such as: node failures, node mobility, less robust to link failures and low reliability. SBMRP finds stable multiple paths from source to destination through the candidate nodes, which have less mobility and more energy, bandwidth and the link quality of a node. Multiple paths are established between source and destination through these candidate nodes and form a routing backbone. SBMRP operates in the following main phases

1. Computation of node mobility, link quality, residual energy and bandwidth of a node using HELLO packet.

2. Selection of nodes as a candidate node, if it has residual bandwidth, residual energy and link quality higher than threshold value and mobility of a node lesser than threshold.

3. Establishing stable multiple paths between nodes using the route discovery technique and constructs routing backbone between the nodes.

4. Route recovery technique takes place against candidate node failure.

The rest of this paper is organized as follows. The next section gives brief description of the existing link or route stability based routing protocols. Design of proposed routing protocol SBMRP is described in section III; finally, the simulation results and conclusions are summarized, respectively in section IV and V.

\section{RELATED WORK}

The description of some work related to link and route stability based routing protocols are given in this section. Based on AODV routing protocol, Sung-Ju Lee and Mario Garcia proposed a new scheme called AODV-BR (Backup Route) [9]. In AODV-BR, the alternative routes are constructed by the RREP packet. Each neighboring node overhears the RREP packets and records the source of one RREP packet as the next hop to the destination in to its alternate route table. In AODV-BR, the establishments of alternate routes rely on the overhearing of routes reply RREP messages. No additional messages are required during the backup routes. AODV-BR can offer more stable connections then AODV can. However, AODV-BR has to pay extra efforts in the maintenance of alternative route table and route recovery.

Ad hoc QoS on demand routing (AQOR) [10] is a reservation based, on demand QoS aware routing protocol based on AODV. It has incorporated the following features for providing QoS- (i) available bandwidth estimation and end-to-end delay measurement, (ii) bandwidth reservation (iii) adaptive route recovery.
Temporary reservation is used to free the reserved resources efficiently at each node when the existing routes are broken. The adaptive route recovery procedure includes detection of broken links and QoS violations and triggering of route recovery at the destination. AQOR has the drawback that, it fails to selects QoS aware with highest stability which is essential to reduce the probability of data path breakage during data transmission.

Flow oriented routing protocol (FORP) was proposed by W. Su and M. Gerla [11], FORP is an on demand routing protocol. FROP uses a global positioning system (GPS) to allow all nodes to get their own information. By using this information, it calculates the link duration time to get the stable path for data transmission. Their proposed scheme introduces a new concept called "multihop handoff" to anticipate topological changes and reduces the rerouting. However, this scheme is effective for discovering long-lived routes, but tends to discover long-hop routes.

Nen-Chung Wang and Shou-Wen Chang [12] have proposed reliable on demand routing protocol (RORP) using mobility prediction technique. The proposed scheme is used to determine the duration between the two connected mobile nodes by using GPS. The proposed RORP selects the path with the longest duration of time for transmission to increase reliability and the longest duration of time is considered as stable route for packet transmission. RORP uses a new mechanism called GPS partial reconstruction process to select a backup path for maintenance. To use this scheme, at least some nodes are required to have GPS devices. So they could pass information for routing. Having GPS like devices is not feasible and unrealistic in most of the ad hoc network environments.

A link availability based QoS - aware (LABQ) routing protocol for MANETs proposed by M. Yu et al. [13]. Proposed protocol designed by the estimation of mobility prediction, link quality measurement and energy consumption to provide high reliable better communication. A proposal by Carofiglio et al. [14] uses path duration the path availability probabilities of link failures caused by node mobility in MANETs by considering the case where the network nodes move according to the random direction mobility model. Using mathematical model, proposed scheme, estimated the probability of path duration till time $t$ and probability of path availability at time $t$. The probabilities of path duration and path availability depend on the mobility pattern of the nodes.

In [15-17], a prediction-based link availability estimation algorithm was developed and investigated with random walk model. The algorithm tries to predict the probability that an active link between two nodes will be continuously available for a certain period $T_{p}$, which is obtained by the simulation results, this algorithm cannot accurately calculate the link availability; it can only reflect the general tendency of the link availability, that is, $L\left(T_{p}\right)$ can approximate $T_{i} / T_{p}$, where $\mathrm{T}_{\mathrm{r}}$ is the mean time that a link will be continuously available corresponding 
to a prediction $T_{p}$. For a given $t$ that is not equal to $T_{p}$, this approach cannot compute the continuous link availability from $\mathrm{t}_{0}$ to $\mathrm{t}_{0}+\mathrm{t}$.

Nen-Chung Wang and Jhu-Chan Chen [18, 19] proposed a stable weight-based on - demand routing protocol (SWORP) for MANETs; weight-based route strategy scheme is used to select a stable route in order to enhance system performance. The weight of a route is decided by three factors: the route expiration time, the error count, and the hop count. Route discovery usually find multiple routes from the source node to the destination node. Then the route selection is based on the weight value of each feasible path. This approach estimates the weight during route discovery; it leads to increase in routing overhead.

In [20] authors designed link expiration time (LET) prediction algorithms to predict the LET between two mobile nodes on an active route. Proposed algorithms are implemented in the DSR algorithm. Three LET prediction algorithms designed based on the i) received signal power ii) GPS and iii) Signal strength. These approaches cannot be suitable because it assumes signal strength. Signal strength can be affected by environmental conditions and its value can change lot for the some node distances. Use of GPS is also critical, because in some environments where the mobile nodes energy is limited the GPS is not functional.

Link availability based routing protocol (LBRP) proposed by Q. Han, Y. Bai, L. Gong, W. Wu [21]. LBRP designed to predict the unpredictable topology change and to reduce frequent link failures. By considered these issues proposed scheme uses analytical model for link availability with random walk mobility model. LBRP gives an accurate estimation of $L\left(d_{0}, t\right)$, which is the probability that the link will be continuously available from $\mathrm{t}_{0}$ to $\mathrm{t}_{0}+\mathrm{t}$ given the initial distance $d_{0}\left(d_{0 \leq}\right.$ $R r$ ). An accurate estimation of $d_{0}$ can be obtained through signal strength.

In [22], the authors have proposed two schemes: AODV with Adaptive Back up Routing (AODV-ABR) and combination of ABR and Local Repair (AODV-ABL) to increase the adaptation of routing protocols to topology changes by modifying AODV-BR. In AODV-ABR, the alternative route can be created by overhearing not only RREP packets but also data packets. AODV-ABL combines the benefits of AODV-ABR and local repair.

In [23] authors proposed an effective and efficient protocol for backup and disjoint path set in an Ad Hoc wireless network. This protocol converges into a highly reliable path set very fast with no message exchange overhead. To provide highly reliable path, estimated LET between each two nodes. The LET value is used as a database to predict the probability of proper operation of links. In their proposed method link reliability obtained by LET and prediction is done by using Multilayer perception (MLP) network. This method predicts the reliability of links between nodes in the ad hoc networks.

Enhanced reliable on demand routing protocol (ERORP), considered the mobility prediction technique to find reliable path based on the RORP Protocol [24].
Proposed scheme uses GPS to obtain the information of each node and this information used for calculating Route Expiration Time (RET). The route with longer RET is considered as stable route. In ERORP during the routing process node calculates the LET. If the LET is greater than the threshold of RET, it forwards the packets else discards the packets .To be more effective, their proposal requires the GPS. In order to use this scheme, at least some nodes are required to have GPS devices. So they could pass on the location information on for routing. Having the GPS devices may not economically viable for many mobile devices.

Nityananda Sarma and Sukumar Nandi [8] and [25] have proposed a Route Stability based QoS Routing (RSQR) protocol in MANETs. Proposed a route stability based QoS-aware routing protocol Uses the following key components. $(i)$ proposed a Route Stability Model (RSM) for computing link stability based on measurements of received signal strengths of two successively received packets from a neighbor. Because, link stability will be in the interval $[0,1]$, used it as the probability of survival of the link in the near future and the route stability is calculated as a product of link stability of all the links which constitute the route under consideration. (ii) A QoS-aware routing with throughput and delay constraints. The routing protocol incorporates the stability model in route discovery process is used to find QoS routes with highest stability. (iii) uses admission control technique during route discovery. (iv) QoS violation detection and recovery.

Lei Geng, Yuebin Bai, Ming Chen [26], developed a mathematical model for link availability estimation with random walk model. This approach gives an accurate estimation of $L\left(d_{0}, t\right)$, which is the probability that the link will be continuously available from $t_{0}$ to $t_{0}+t$ given the initial distance $d_{0}\left(d_{0 \leq} R r\right)$. An accurate estimation of $d_{0}$ can be obtained through GPS. In [27] authors used analytical model to estimate the link availability between the nodes.

In [28] authors designed scalable routing protocol called link-stability and energy aware routing protocols (LAER) based on joint metric of link stability and energy drain rate has been proposed. link- stability aware metric and energy aware metric are designed using the multi objective objective mathematical formulation model. In [29], authors proposed a routing strategy that modifies the route request (RREQ) packet of the AODV routing protocol. The proposed protocol uses the threshold value of residual battery to transfer the data packets, if the threshold value is below the threshold value, a node is not allowed to relay data/control packets.

The above referred link or route stability and reliable based routing protocols proposed to improve link quality, routing efficiency and QoS. Most of the proposed link or route stability based routing protocol designed using the prediction algorithms [15-11], [22], some of the existing routing protocol [11], [12], [21], [26-28], [30] uses LET, RET to estimate the stability metric. These techniques relay on the special devices, such as GPS to detect the exact position of the mobile nodes. In order to use such 
schemes, at least some nodes are required to have GPS devices. So, they could pass on the location information for routing. However, Having GPS like devices may not be economically viable, feasible and unrealistic in most of the ad hoc network environment. There have been many protocols designed by considering the signal strength [8], [31]. However, this approach is cannot be suitable because it assumes that the signal strength can be affected by environmental conditions and its value can change a lot also for some nodes distance. This determines many fluctuations in the radio signal measurement, producing erroneous considerations on the link stability.

Some of the existing schemes use backup paths and local route repair technique [19], [25]. These schemes can be effective before the path breaks, once the path breaks this is not useful. Some backup based routing protocols are designed, backup routing schemes needs extra space to store and maintain the backup routing information and backup routes may solve the route failures problem temporary and is not effective in QoS aware data transmission. In some of the existing routing protocol, Link quality and residual energy of each node is estimated during the routing process, results increased delay and overhead. The main drawback of the existing work is either usage of unreliable parameters which are prone to errors and variations. The proposed work is motivated by observing the drawback of existing protocols.

\section{DESIGN OF SBMRP}

\section{A. Overview}

In this paper, we construct a stable backbone based multi-path routing protocol. Backbone is a set of candidate nodes that are part of the network which have some special dedicated properties. Candidate nodes are selected depending on residual bandwidth, residual power, mobility and link quality. In the first phase, nodes exchanges HELLO packets containing the above parameters. Then the nodes with high residual bandwidth, residual power, link quality and low mobility are designated as candidate nodes. In the second phase, multiple paths are established between each source and destination through these candidate nodes, thus forming a routing backbone. So the candidate nodes become multipath enabled nodes which can transfer data using multipath links. If a source broadcasts a route request, then route is established from the source to destination via the backbone candidate nodes and data is transmitted. If any candidate node in the path tend to fail due to lack of bandwidth, residual energy or link quality, alternate path through another candidate node can be established. Thus the candidate nodes from the routing backbone are rotated minimizing their overhead and energy.

\section{B. Estimation of metrics}

\section{1) Estimation of residual bandwidth (RBW)}

Each node that wants to transmit the data has to be aware of its local bandwidth and its neighboring nodes information within the interference range. As bandwidth is shared among neighboring nodes, the node pays attention to the channel and estimates local bandwidth $(B W L)$ It depends on the ratio of idle and busy time period for a predefined interval. [30]

$$
B W L=C_{C H} *\left(T_{i} / T_{i n}\right)
$$

Where $C_{C H}$ is the channel capacity, $T_{i}$ is the idle time period in the predefined time period $T_{i n}$. The minimum bandwidth (BWmin) of all the nodes with the interference range is identified as the result of prior collection of neighboring node information. Hence the difference among $B W$ min and $B_{L}$ gives the $R B W$ of the node.

$$
R B W=B W L-B W \min
$$

\section{2) Estimation of residual energy $\left(E_{r}\right)$}

Let $E_{i}$ be the initial energy of a node. After the time period $\mathrm{t}$, the energy consumed by the node $(E(t))$ is given using following equation

$$
E(t)=n_{t x} * \varepsilon+n_{r x} * \delta
$$

Where, $n_{t x}$ and $n_{r x}$ are the number of data packets transmitted and received by the node after time $t$. $\varepsilon$ and $\delta$ are constants in the range $(0,1)$. The $E_{r}$ of a node at time $\mathrm{t}$ is computed using the following equation.

$$
E_{r}=E_{i}-E(t)
$$

\section{3) Estimation of node mobility $\left(M_{j}^{i}\right)$}

The mobility of the node $j$ with respect to node $i\left(\mathrm{M}_{\mathrm{j}}^{\mathrm{i}}\right)$, is estimated based on the ratio of received signal strength (RSS) among the two consecutive packet transmissions from a neighbor node [31]. It is given using the following equation.

$$
M_{j}^{i}=10 \log 10 \frac{R S S_{i \rightarrow j}^{\text {new }}}{R S S_{i \rightarrow j}^{\text {old }}}
$$

Where,

$$
R S S=\beta * \vartheta * P_{t x}
$$

$\beta$ is constant that depends on the wavelength and the antennas, $\vartheta$ is the channel gain and $P_{t x}$ is the Signal power of the transmitter.

\section{4) Estimation of link quality $(L Q)$}

$L Q$ is defined as the ratio of number of error bits (Berror) to the number of received bits (Btx) [32].

$$
L Q=\text { Berror } / \text { Btx }
$$


Every time when the node receives the data packet the above computed value gets updated. This value depends on the interference effect of the wireless channel, additive white Gaussian noise and signal transmission range.

\section{Packet Formats used}

\section{1) HELLO Packet}

HELLO packet used to select candidate node. Before we execute the route discovery process, we need to find candidate node. To find the candidate nodes, source node sends a HELLO message to neighboring nodes. The purpose of the HELLO messages is to find the links of the neighboring nodes and to select the candidate nodes. Packet format of HELLO message is shown in table 1. It includes the parameters such as the parameters such as $R B W, E_{r}, M_{j}^{i}$ and $L Q$.

Table 1. Hello Packet Format

\begin{tabular}{|l|l|l|l|l|}
\hline Node ID & RBW & $\mathrm{E}_{\mathrm{r}}$ & $\mathrm{M}_{j}^{i}$ & LQ \\
\hline
\end{tabular}

Where, Node Id indicates the node identifier. Node number is considered as node identifier. $R B W$ is the residual bandwidth of a node required to transmit to transfer the packet. $E_{r}$ is the residual energy of a node that is used to transmit and receive the packet. $M_{j}^{i}$ indicates the mobility of the node $j$ with respect to the node $i . L Q$ is the ratio of number of bits to the number of received bits. The node information such as $R B W, E r, L Q$ and $\mathrm{M}_{\mathrm{j}}^{\mathrm{i}}$ are stored in the node information table (NIT).

\section{2) Route Request (RREQ) Packet}

When a source node wants to transfer the packets and if it has no routing information in its routing table, it sends a RREQ packet to its neighboring nodes. The following table shows the route request packet format.

Table 2. RREQ Packet Format

\begin{tabular}{|c|c|c|c|c|}
\hline Src ID & Dest ID & $\begin{array}{c}\text { Previous } \\
\text { hop node ID }\end{array}$ & $\begin{array}{c}\text { Previous } \\
\text { Nodes } \\
\text { State }\end{array}$ & $\begin{array}{c}\text { Nodes } \\
\text { State }\end{array}$ \\
\hline
\end{tabular}

Where, Src. ID is the address of the node originating the packet, Dest. ID is the address of destination, Prev. node Id is the address of the previous node. This field is updated after every movement to the next node until it reaches the receiver. Prev. node state indicates the previous node state. They are used to trace out the candidate node. In case a node is not a candidate node, by looking at this field, we can determine whether its previous node is candidate node or not. Node state field intimates whether the node is designated as candidate node or non-candidate node.

\section{Stable Backone-based Multipath Rouring Protocol}

SBMRP includes two phases: First, the selection of reliable and efficient nodes from a set of mobile nodes in the networks. The reliable nodes are designated as candidate nodes because candidate nodes have some special dedicated properties and the candidate nodes are selected depending on the $R B W, E r, M_{j}^{i}$ and $L Q$. Second the construction of a routing backbone; backbone is a set of candidate nodes.

\section{1) Selection of Candidate Node}

The steps involved in the construction of backbone are as follows.

1) When the nodes are deployed in the network, it initially broadcasts HELLO messages to its neighbors.

2) Based on the HELLO Message, each node identifies itself and also maintains the neighbors list (NL).

3) Based on the obtained parameter values (Estimated in section 3.2.1-3.2.4), each node analyzes the following condition and when the condition is satisfied it is designated as candidate nodes.

$$
\begin{aligned}
& \text { If }(R B W>T h(R B W)) \&\left(E_{r}>T h(E r)\right) \&\left(M_{j}^{i}<T h\right. \\
& \left.\left(M_{j}^{i}\right)\right) \&(L Q>T h(L Q)) \\
& \text { Then }
\end{aligned}
$$

\section{Node is declared as Candidate} End if

An example of candidate node selection is shown in the fig. 1 for an arbitrary network with 20 nodes. The candidate node selection process starts by broadcasting the HELLO packets to its neighbors that calculates the $R B W, E_{r}, M_{j}^{i}$, and $L Q$ and these values is stored in the NIT. Based on the threshold values of bandwidth, energy, mobility, link quality and information stored in the node information table, the nodes 5, 6, 8, 9, 10, 11, 12, 13, 14, 15 and 19 contains the maximum energy, bandwidth and link quality and with minimum mobility. So, these nodes are chosen as candidate nodes.

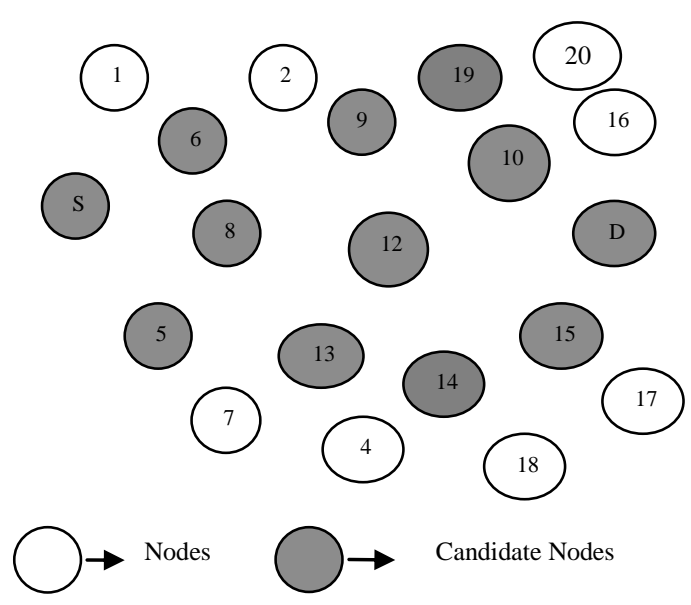

Fig.1. An Example of Selection of Candidate Nodes.

\section{2) Costruction of Routing Backbone}

The second phase of SBMRP is the construction of routing backbone, this phase includes two techniques one is route discovery and the other is route recovery process. 


\section{a) Route Discovery}

The SBMRP is a reactive routing protocol and the route discovery process is similar to that of other on demand routing protocols in MANETs. The route discovery process starts when a source node needs to transfer the packets to the destination. SBMRP is a multipath routing protocol; it discovers multiple node disjoint paths from source to destination on a single route discovery process. To start the route discovery, the source node floods the RREQ packet to its neighboring nodes. The RREQ packet format is shown in the table 2 .

The detailed route discovery process is explained in the following steps.

Step 1. The source node $S$ broadcasts the RREQ message to its neighboring nodes. If the node is within the transmission range, it forwards the RREQ packet to its own neighboring nodes and adds its ID, Previous hop node ID, previous Node State and Node State.

Step 2. When $N_{i}$ receives $R R E Q$ updates its routing table with the information that includes source $I D$, destination ID, previous hop node ID and its state. It appends its state to the node state field to RREQ message and analyzes the destination ID.

If $N_{i} \neq D$

Then

$N_{i}$ rebroadcasts $R R E Q$ to neighboring nodes

Else

Ni sends RREP to Source Node.

End if

The above condition intimates that when intermediate comes to know that it is destination node, it sends RREP message to source. Otherwise, it rebroadcasts RREQ to its neighboring nodes.

Step 3. In case Ni receives two or more RREQ with similar destination ID, then it considers first received message and discards the other message.

Step 4. When $D$ receives $R R E Q$ message, it appends its state to RREP and unicast the reply message in the reverse path towards $S$. D performs this similar action for every $R R E Q$ it receives.

Step 5. Ni upon receiving the RREP message appends its state to the message and also updates its routing table, it unicasts the RREP in the direction of $S$ utilizing the previous hop node information which is priory stored.

Step 6. Step 5 is repeats till RREP reaches to the source node $S$.

Step 7. Then $S$ establishes the multiple paths from $S$ to $D$ through the candidate nodes and $S$ chooses the path with candidate nodes to transmit the data packet.

The fig. 2 illustrates an example of the basic operation of route discovery phase. When the source node (S) wants to transfer the data packets, $S$ broadcasts RREQ packet to discover path to destination $D$. When the intermediate nodes $6,8,5$ receives the RREQ it updates the routing table information and appends the node state field then node $6,8,5$ then broadcasts the RREQ packet to its neighboring nodes. On receiving the RREQ packet, destination node D uncast RREP packet and setup the reverse path towards $S$. Then, the multiple routes established between $\mathrm{S}$ to $\mathrm{D}$. The set of candidate nodes forms multiple paths; these paths are considered as a backbone for routing. The routes are S-6-9-10-D, S-8-12$\mathrm{D}$ and S- 5-13-14-15-D is established from source $\mathrm{S}$ to destination D.

\section{b) Route Recovery Technique}

When the candidate node fails then it triggers the route recovery technique, before route breaks. If candidate node fails, candidate node broadcasts the route error packet (RERR) to its neighbor nodes. on receiving the REER packet, neighboring nodes check its routing table and finds the alternative path through the candidate node from the established path, before path breaks. When all established path fails, it initiates the route rediscovery.

The steps involved in route recovery technique are

Step 1. If $(R B W<T h(R B W))\left\|\left(E_{r}<T h(E r)\right)\right\|\left(M{ }_{j}^{i}>\right.$ $\left.\operatorname{Th}\left(M_{j}^{i}\right)\right) \|(L Q<T h(L Q))$

Step 2. Broadcasts the REER packet to the sending node. $O$ n receiving a REER packet, it applies the route recovery technique and node finds an alternative path from the other candidate node or by the established path. The candidate node acts as a back bone of the protocol.

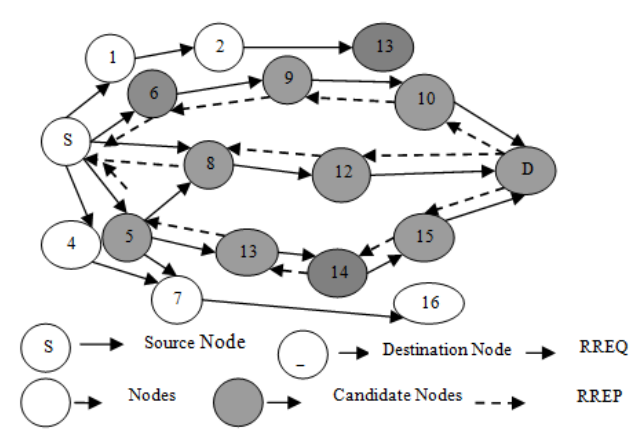

Fig.2. An Example of Route Discovery Process

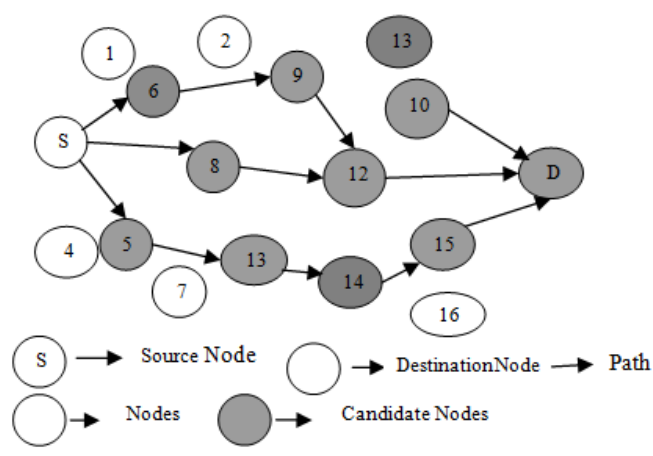

Fig.3. An Example Of Route Recovery Process 
Fig. 3 shows an example of route recovery process, candidate node 10 fails because of the residual energy is reduced below the minimum threshold. This in turn affects the data transmission and breaks the path from $9-$ 10. In order to overcome this problem the SBMRP initiates the route recovery that is an alternate path via candidate node 12 is chosen to transmit the data.

\section{Simulation SETUP AND PERFORMANCE ANALYSIS}

In this section, compare the performance of proposed protocol SBMRP with AODV, AOMDV and RSQR under various scenarios by simulation method.

\section{A. Simulation Parameters}

The simulation is performed using the network simulator (NS - 2) [33]. The simulated environment consists of 100 wireless nodes placed randomly in a 1000 $\mathrm{x} 1000 \mathrm{~m}^{2}$ area with varied speed from $5-100 \mathrm{~m} / \mathrm{s}$, random waypoint mobility model was used to place the nodes randomly. The pause time is varied from 0-100 s. Each node randomly selects a position and moves towards that location with a speed between minimum and maximum speed. Once it arrives at the position, it stays for predefined time, after that time, it results a new position and repeats the process. The transmission range was assumed as $250 \mathrm{~m}$ and the channel capacity is $2 \mathrm{Mbps}$. The two ray propagation model was assumed, constant bit rate (CBR) traffic model is used. Each node uses a drop tail queue and holds maximum of 50 packets at the constant size of 512 bytes. The simulation is performed for 100s. The simulation parameters are summarized in table 3 .

Table 3. Simulation Parameters

\begin{tabular}{ll}
\hline \multicolumn{1}{c}{ Parameter Name } & \multicolumn{1}{c}{ Value } \\
& \\
No. of nodes & 100 \\
Rate & $250 \mathrm{~kb}$ \\
Network size & $1000 \mathrm{x} 1000 \mathrm{~m}^{2}$ \\
MAC Protocol & IEEE $802.11 \mathrm{DCF}$ \\
Channel capacity & $2 \mathrm{Mbps}$ \\
Transmission range and carrier sense & $250 \mathrm{~m}$ \\
range & \\
Simulation time & $100 \mathrm{sec}$ \\
Packet size & $512 \mathrm{bytes}$ \\
Nodes speed & $5-100 \mathrm{~m} / \mathrm{s}$ \\
Pause time & $0-100 \mathrm{~seconds}$ \\
Antenna type & Omini Antenna \\
Minimum bandwidth & $40 \mathrm{kbps}$ \\
Initial energy (E) & $5 \mathrm{~J}$ \\
$\mathrm{E}_{\mathrm{r}}$ and $\mathrm{E}_{\mathrm{t}}$ & $0.5 \mathrm{~nJ} / \mathrm{bit}$ \\
Th (RBW) & $0.5 \mathrm{~nJ} / \mathrm{bit}$ \\
Th (E $\mathrm{E}_{\mathrm{R}}$ ) & $8 \mathrm{Kbps}$ \\
Number of runs & $5 \mathrm{~J}$ \\
Routing protocols & $\mathrm{AODV}$, \\
& $\mathrm{RSQR}, \mathrm{SBMRP}$ \\
& \\
\hline &
\end{tabular}

\section{B. Performance Metrics}

The following metrics are used to evaluate the performance of the protocols. Our objective is to increase the Packet delivery ratio (PDR) and reduce the overheads (like control, memory and computational overheads) and packet delays. Increased PDR and reduced packet delay improves network throughput and reduced overheads reduce bandwidth consumed and efficient usage of various resources for route discovery and maintenance.

1) PDR: It is the ratio of the number of data packets received successfully by the destination to the number of data packets actually sent by the corresponding source in the network. It is used to measure the link quality of discovered path.

2) End-to-end delay: End-to-end delay is the time taken to transfer the packet from source node to the destination node.

3) Normalised Routing Load: It is defined as the ratio of the number of control packets generated to the total number of data packets generated by the source.

4) Packet drop ratio: It is defined as the number of packets dropped during the data transmission of data packets from source node to the destination.

\section{Performance Analysis}

This section gives the performance analysis of proposed SBMRP with that of AODV [12], AOMDV [14] and RSQR [16]. In [34], [35], compared the performance of RSQR and SBMRP with varying mobility speed and pause time respectively. In this paper, the performance of SBMRP is compared with traditional RSQR, unipath routing protocol (AODV) and multipath routing protocol (AOMDV). For the performance analysis, conducted test on two cases, first, network with 100 mobile nodes topology by varying pause time from $0-100$ s and another case is network with 100 mobile nodes topology by varying speed from $5-100 \mathrm{~m} / \mathrm{s}$. Fig. $4-11$ shows the relative performance of SBMRP, AODV and AOMDV by varying pause time and mobility speed respectively. As is expected, the performance of the routing protocols increases as the pause time increases and performance of the routing protocols degrades as the speed increases. This is because the longer pause time implies less mobility and higher speed implies high mobility.

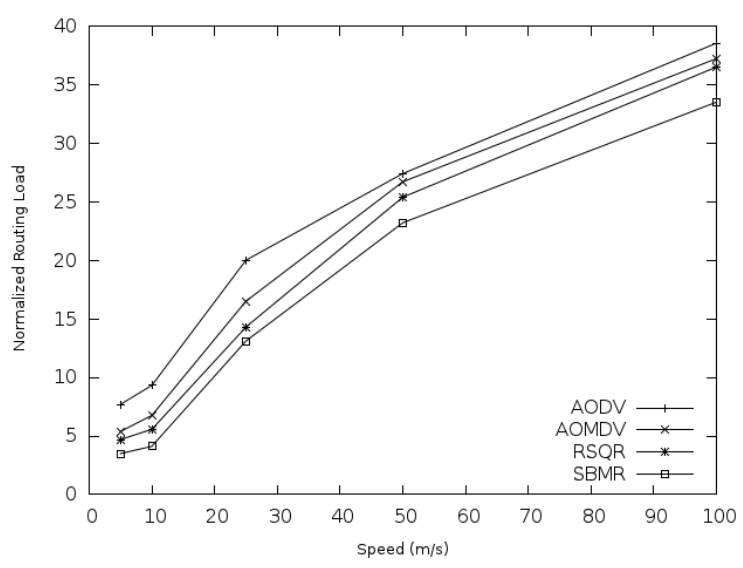

Fig.4. Normalized Routing Load against Node Speed 
Normalized routing load by varying pausetime and speed is shown in fig. 4 and 5 respectively. As we can see from the fig. 4 , the routing load is decreased as the pause time increased this is due to less variations of network topology. When the pause time increases, the link failures caused by node mobility will also reduces which implies the primary route is stable. Therefore, no matter what routing scheme is used, the routing load is decreased. The routing load included the RREQ, HELLO, RREP and RERR packet overhead.

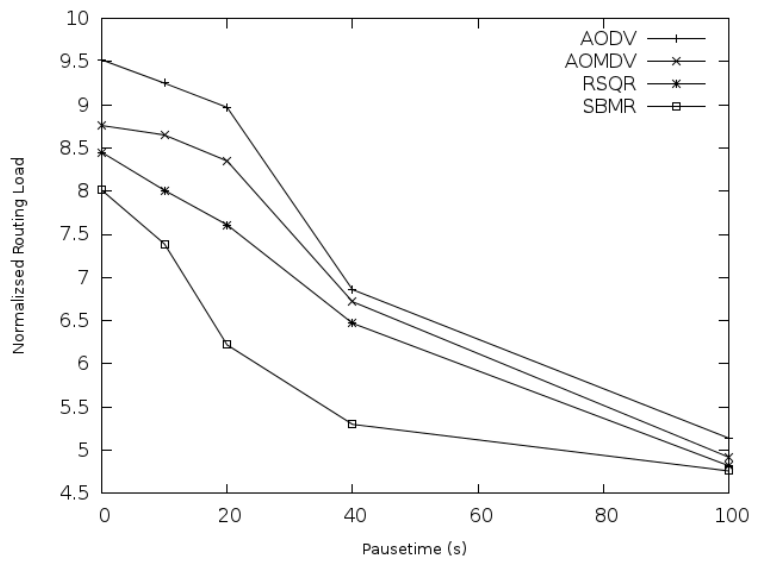

Fig.5. Normalized Routing Load against Pause Time

From Fig. 5, we can see that the routing load increases as the mobility speed increases. When the speed of the mobile node increases the mobility of node increases, the routing path was more unreliable the reason is that there were more chances for routes to break and leads to route rediscovery. Route rediscovery rebroadcasts the more control packets. Thus, the routing load increased. The normalized routing load of proposed scheme SBMRP is less than that of AODV, AOMDV and RSQR. This is because SBMRP uses the more stable route to forward the packets while the AODV and AOMDV use the shortest path or fastest path for data transmission. A shortest path leads to more failures in paths. The routing load of RSQR is less then AODV and AOMDV. Because, the RSQR uses the route stability model for data transmission. SBMRP routing load is less then RSQR because of less route rediscovery. The reason for that are i) SBMRP uses the backbone based multipath routing for data transmission before path breaks, ii) SBMRP uses less route rediscovery because it uses the route recovery technique, iii) SBMRP finds the multiple stable paths in a single route discovery. So, from figs. 4 and 5 it is clear that our proposed protocol obtain less overhead when compared with the existing protocols.

Figs. 6 and 7 show the results of PDR with varying pausetime and speed respectively for the four protocols namely, SBMRP, AODV, AOMDV and RSQR. From the results shown in figs. 6 and 7 , we can observe that PDR of SBMRP improves significantly in comparison with RSQR, AODV and AOMDV protocols. The PDR of SBMRP increases as the pause time increases and a decrease as the speed increases, reason is mobility. From fig. 6 we have seen that SBMRP PDR increases as the pausetime increases. PDR of SBMRP is more than $91 \%$ in all scenarios and PDR of AODV, AOMDV and RSQR is more than $81 \%, 86 \%$ and $89 \%$ respectively. Fig. 7 shows the PDR with different mobility speeds. We can see that, the mobility of a node increases PDR of routing protocols are decreased. The reason is that the routing path was very easy to break.

From figs. 6 and 7 we have seen that the PDR of RSQR is more than AODV and AOMDV, because RSQR routing protocol uses the route stability model, it finds the stable path. Our proposed scheme SBMRP shows the better performance for both test cases with varying pause time and speed. This is due to i) SBMRP uses the stable path through the candidate nodes to forward the packets, ii) SBMRP uses backbone paths iii) SBMRP finds the alternate path to transfer the data packets before path breaks.

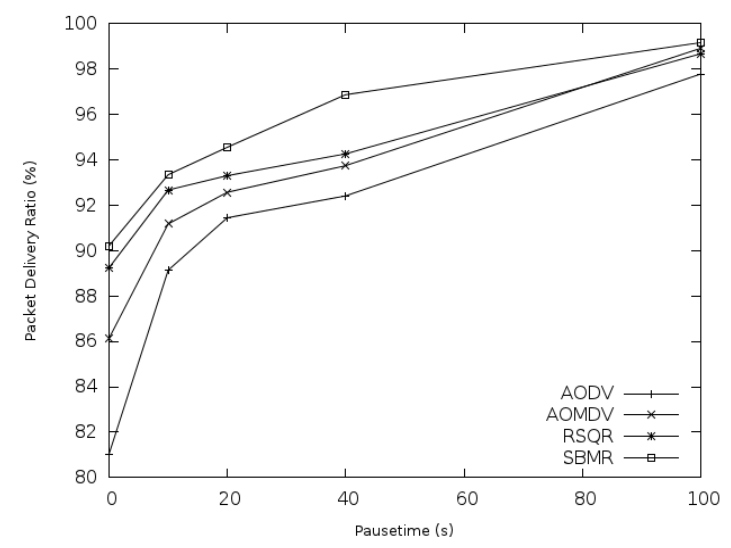

Fig.6. PDR against Pause Time

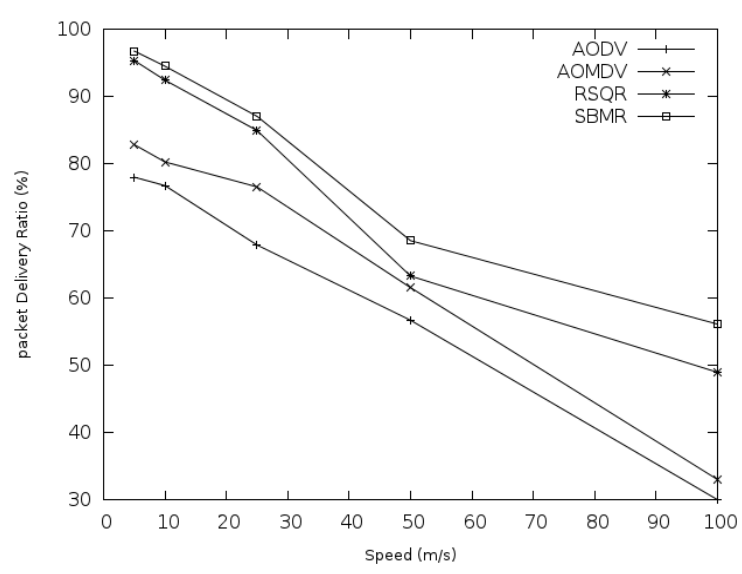

Fig.7. PDR against Speed.

Figs. 8 and 9 shows comparison of packets drop ratio for the SBMRP, AODV, AOMDV and RSQR for different pause time and mobility respectively. From the figs. 8 and 9 we can see that packet drop ratio decreases as the pause time increases and decreases as speed decreases. The reason is due to less and mobility. In both cases the number of packets dropped by the SBMRP is lower than the AODV, AOMDV and RSQR. This is due to the use of strong backbone nodes and multipath routing for forwarding the data. 


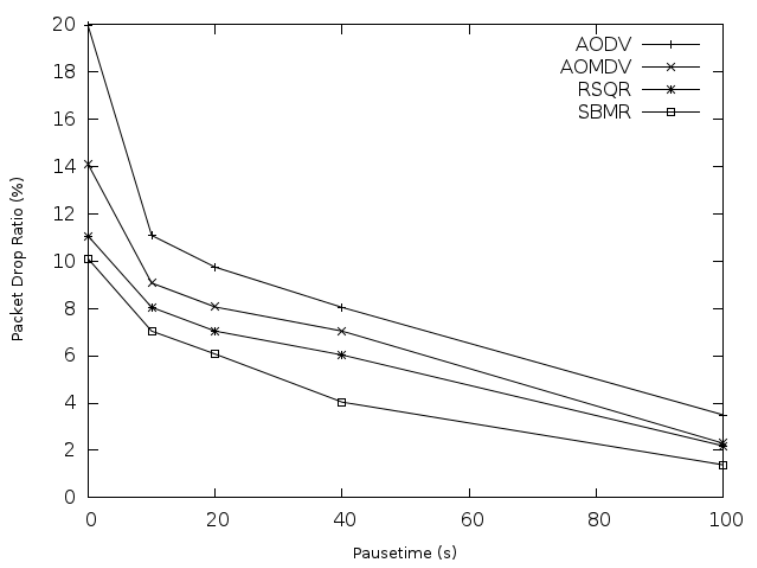

Fig.8. Packet Drop Ratio against Pause Time

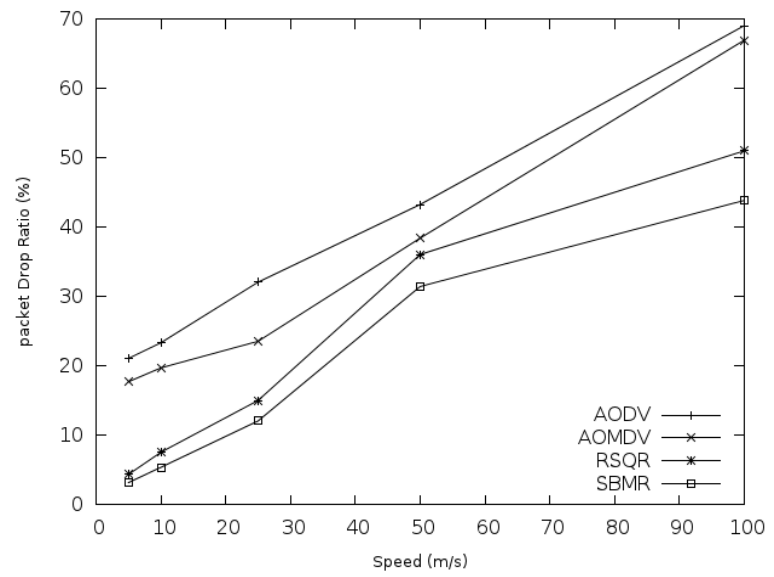

Fig.9. Packet Drop Ratio against Speed.

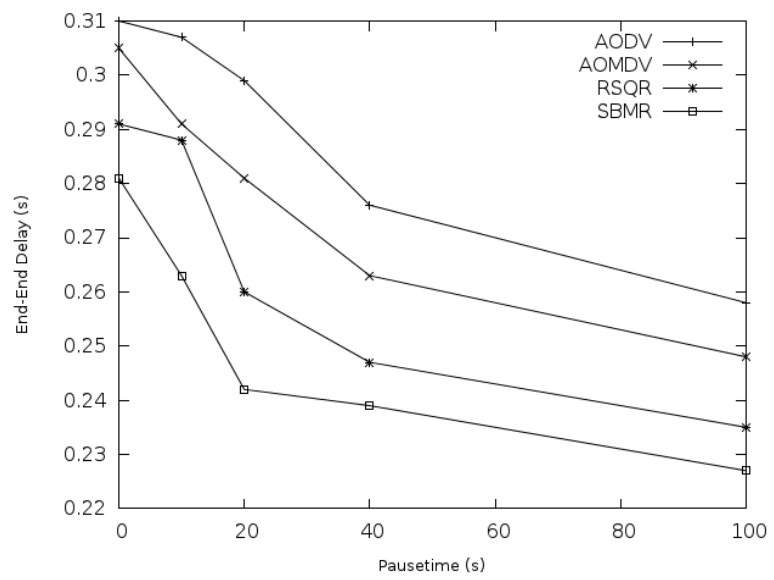

Fig.10. End - end Delay against Pause Time

Figs. 10 and 11 show the end-end delay of AODV, AOMDV, RSQR and SBMRP for different pause time and speed, respectively. End - end Delay increases as the mobility increases and decreases as the pause time increases. From the fig. 11, it can be seen that end - end delay is less $0.031 \mathrm{~s}$ for all the protocols in all scenarios. End-end delay of our proposed scheme SBMRP is less compared to AODV, AOMDV and RSQR. The reason, SBMRP uses the stable multiple paths for data transmission and SBMRP uses route recovery technique before path breaks. This reduces the route rediscovery.

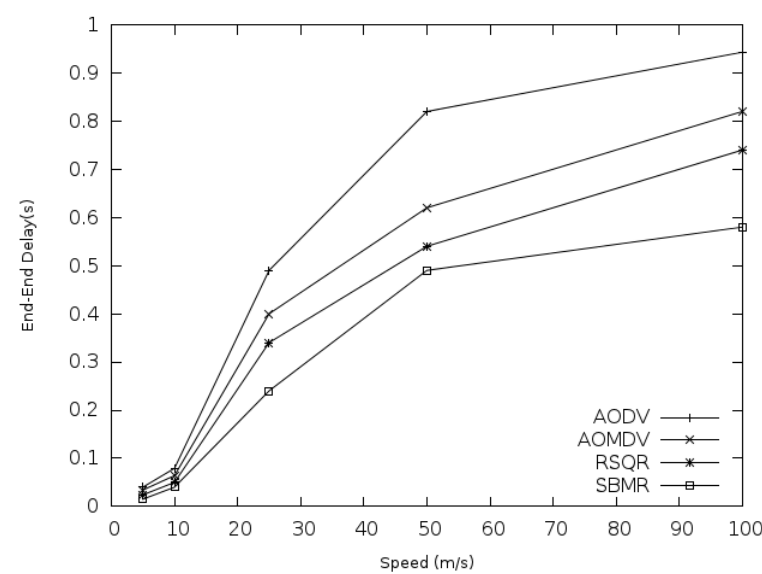

Fig.11. End - end Delay against Speed

From the above analysis, we conclude that proposed scheme outperforms the AODV, AOMDV and RSQR and shows significant performance improvement in dynamic network environments and shown that the SBMRP proposed technique minimizes the packet drop, delay and overhead while maximizing the PDR under high mobility.

\section{CONCLUSION}

In this paper, we have constructed a stable backbone based on demand multipath routing protocol (SBMRP) for MANET. Initially, nodes with high $R B W, E r, L Q$ and low $M_{j}^{i}$ are designated as candidate nodes. Then the multiple paths are established between each source and destination through these candidate nodes, thus forming a routing backbone. So, the candidate nodes become multipath enabled nodes which can transfer data using multipath links. If any candidate node in the path tend to fail due to lack of bandwidth, $E r$ or $L Q$, alternate path through another candidate node can be established. Simulation result shown that SBMRP was able to locate more stable paths then the RSQR. So, the successful delivery rates of packets SBMRP were higher than the RSQR, AODV and AOMDV, the packet drop and endend delay of SBMRP is smaller than the other routing protocols. Because of less route discovery the overhead of SBMRP is less than the RSQR, AOMDV and AODV. In future works, we plan to take up the mathematical analysis of the in which we wish to compare the analytical results with simulation results.

\section{REFERENCES}

[1] C. Siva Ram Murthy and B. S. Manoj, "Chapter - 3 Ad Hoc Wireless Networks", in Ad Hoc Wireless Networks: Architectures and Protocols, (Prentice Hall, june 2000, 1st Edition), pp. $213-245$.

[2] http://www.ietf.org/html.chapters/manet-chapter.html.

[3] P. Johnson, "Routing Protocols for Mobile Ad-Hoc Networks - a comparative performance analysis", Proc. ACM/IEEE Int. Conf. on Mobile Computing and Networking (MobiCom'99), Seattle, WA, Aug. 15-19, 1999, pp. 195-206. 
[4] C. E. Perkins and E. M. Royer, "Ad-Hoc on-demand distance vector routing", Proc. Second IEEE Workshop on Mobile Computing Systems and Applications, New Orleans, Louisiana, USA, 25-26 February 1999, pp. 90100.

[5] D. Johnson and D. A. Maltz, "Dynamic Source Routing Protocol in Ad Hoc Wireless Networks", Internet Draft, drfat-ietf-manet-dsr-10.txt, july 2006.

[6] V. D. Park and M. S. Corson, "A highly adaptive distributed routing algorithm for mobile ad- hov networks", Proc. Conference on Computer Communications, Sixteenth Annual Joint Conference of the IEEE Compter and Communications Societies, Driving the Information Revolution (IEEE INFOCOM 1997), Kobe, Japan, April 7-12, 1997, pp. 1405-1413.

[7] Marina, M. K. and Das, S. R , "On -demand Multipath Distance Vector Routing in Ad Hoc Networks", Proc. 9th International Conference on Network Protocols (ICNP 2001), Riverside, CA, USA, 11-14 November 2001, pp. 14-22..

[8] Nityananda Sarma and Sukumar Nandi, "Route Stability Based QoS Routing in Mobile Ad Hoc Networks", Springer Wireless Personal Communications, Vol. 54, 2010, pp. 203-224.

[9] L. S Lee, M. Gerla., "AODV-BR: Backup routing in Ad Hoc networks", Proc. of IEEE Conf. Wireless Communication and Networking (WCNC 2000), Chicago, 23-28 Sep. 2000, pp. $1311-1316$.

[10] Qi Xue and Aura Ganz, "Ad hoc on-demand QoS routing in mobile ad hoc networks", Elsevier journal of Parallel Distrb. Comput. , Vol. 6, 2003, pp. 154-165.

[11] W. Su, and M. Gerla. , "IPv6 flow handoff in ad hoc wireless networks using mobility predication", Proc. IEEE GLOBECOM, 5-9 December 1999, pp. 271-275.

[12] N. C Wang and J. C Chen, "Reliable on demand routing protocol for mobile ad hoc networks with mobility prediction", Elsevier journal of computer networks, Vol. 16, 2007, pp. 823-831.

[13] Aniket Malvankar, Wei S and Simon Y. Foo, "A link availability-based QoS-aware routing protocol for mobile ad hoc networks", Elsevier journal of computer communications , 29, 2009, pp. 123-135.

[14] Givanna Carofiglio, Michele Garetto, "Route Stability in MANETs under Random Direction Mobility Model", IEEE transactions on Mobile Computing, Vol. 8, 2009, pp. $1167-1179$.

[15] He, D. J, Jiang, S. M. and Rao, J. Q., “A Prediction-Based Link availability Estimation for wireless ad hoc networks". Proc. IEEE INFOCOM 2001, Anchorage, 21-26 April 2001, pp. 1745-1751.

[16] S. M Jiang, "An Enhanced Prediction-Based Link availability Estimation for wireless ad hoc networks", IEEE Transactions on Communications, Vol. 52, 2007, pp. 183-186.

[17] D. H. He, S. M Jiang, and J. Q Rao, "A Prediction-Based Link availability Estimation for wireless ad hoc networks", IEEE Transactions on Networking, Vol.13 2006, pp. $1302-1312$

[18] N. C Wang and J. C Chen, "A Stable On-Demand Routing Protocol for Mobile Ad Hoc Networks with Weight-Based Strategy", Proc. IEEE Seventh International Conference on Parallel and Distributed Computing, Applications and Technologies (PDCAT 2006), Taipai, 4-7 Dec. 2006, pp. 166-169.

[19] N. C Wang and J. C Chen, "A Stable On-Demand Routing Protocol for Mobile Ad Hoc Networks with Weight-Based Strategy", Elsevier journal on Information
Sciences, Vol. 177 , 2007, pp. 5532-5537.

[20] Rhim, Achour and Dziong, Zbigniew, "Routing Based on Link Expiration Time for MANET Performance Improvement", Proc. IEEE Malaysia International Conference on Communication, Malaysia, 15-17 Dec 2009, pp. 555-560.

[21] Q. Han, Y. Bai and L. Gong, "Link availability prediction-based reliable routing for mobile ad-Hoc Networks", IET Communications, Vol. 5, 2007, pp. 22912300.

[22] Wei Kung Lai, Sheng yu hsiao and Yuh-chung Lin, "Adaptive Backup Routing for Ad- Hoc Networks", Elsevier Journal of Computer Communication, pp. 30, 2007, pp. 453-464.

[23] Arash Dana, Ahmad Khadem Zadeh and Syeda Ali Sadat Noori, "Backup Path Set Selection in ad-hoc wireless network using link expiration time", Elsevier Journal of Co mputers and Electrical Engineering, Vol. 34, 2008, pp. 503-519.

[24] Fatemeh Razani and Dr. Hassan Taheri, "Enhanced Reliable On-Demand Routing Protocol", Proc. $3^{\text {rd }}$ inter. Conf. on computer science and Technology (ICCSIT 2010), Chengdu, 9 - 11 July 2010, pp. 342-346.

[25] Nityananda Sarma and Sukumar Nandi, "Multipath QoS Routing with Route Stability in Mobile Ad Hoc Networks", IETE Technical Review, Vol. 27, 2010, pp. $380-391$.

[26] Lei Gong, Yuben Bai and Chen, Ming. "Link Availability Prediction in Ad Hoc Networks", Proc. of 14 IEEE international conference on Parallel and Distributed Systems, 2008, pp. 423-428.

[27] Lei Gong, Yubein Bai and Ming Chen, "A Novel Approach of Link Availability Prediction in Ad Hoc Networks". Proc. of IEEE international conference, pp. 26-30, 2008.

[28] De Floriano Rango, Francessca Guerriero and Fazio, Peppino, "Link-Stability and Energy Aware Routing Protocol in Distrubuted wireless Networks", IEEE Transcations on Parallel and Distrubuted Systems, Vol. 23, 2012, pp. $713-726$.

[29] Prem Chand, Dr. M.K.Soni "A Novel Routing Scheme for Mobile Ad Hoc Network", I. J. Computer Network and Information Security, Vol. 4, pp. 17-24, 2013.

[30] Tung-Shih Su, Chih-Hung Hsieh Lin and Wen-Shyong, "A Novel QoS-Aware Routing for Ad Hoc Networks", Proc. of the 9th Joint Conference on Information Sciences (JCIS), 2006.

[31] Vinay Rishiwal, S. Verma, and S. K. Bajpai, "QoS Based Power Aware Routing in MANETs", International Journal of Computer Theory and Engineering, Vol. 1, 2009, pp. 47-57.

[32] P. Basu, N. Khan and D. C Little, "A Mobility Based Metric for Clustering in Mobile Ad Hoc Networks", Proc. 21 st International Conference on Distributed Computing Systems Workshops (ICDCS 2001 Workshops), Phoenix, AZ, USA, 16-19 April 2001, pp. 413 - 418.

[33] Rajashekhar Biradar, Sunilkumar Manvi, Mylara Reddy, "Mesh Based Multicast Routing in MANET: Stable Link Based Approach", International Journal of Computer and Electrical Engineering, Vol. 2, 2012. http://www.isi.edu.nsnam/ns, 2013.

[34] Sujata V Mallapur and Siddarama R Patil, "Stable Backbone based Multipath Routing Protocol for Mobile Ad Hoc Networks", IEEE ICCPCT 2013, January 2013. pp. 1105-1110.

[35] Sujata V. Mallapur and Siddarama R Patil, "Route Stability Based on Demand Multipath Routing Protocol 
for Mobile Ad Hoc Networks", IEEE third International Conference on Communication and Signal Processing, April 2014, pp. 1288-1292.

\section{Authors' Profiles}

Sujata V Mallapur is a $\mathrm{Ph}$. D student at Visvesvaraya Technological University (VTU) doing research in the field of designing stability based routing protocol for mobile ad Hoc Networks. She received BE (Information Science \& Engineering) and $\mathrm{M}$. Tech (Computer Science \& Engineering) degrees from Poojya Doddappa College of Engineering, Gulbarga under VTU, Belgaum in 2004 and 2008 respectively. Her research includes Mobile Ad Hoc Networks, Vehicular Ad Hoc Networks.

Siddarama R Patil received B.E. degree in Electronics and Communication Engineering from Gulbarga University, the $\mathbf{M}$. Tech in Telecommunication Engineering., and $\mathrm{Ph}$. D from
Indian Institute of Technology, Khargpur, India in 1999 and 2009 respectively. Currently, he is Professor and Head in Electronics \& Communication and Engineering Department at Poojya Doddapa Appa College of Engineering, Gulbarga, India. $\mathrm{He}$ has published more than 20 research papers in various national and international journals and conferences. He has guided many B.E. and M. Tech Projects, and guiding more than $8 \mathrm{Ph}$. D students. His current research includes Information Theory and Coding, Turbo Codes, LDPC codes, Iterative decoding algorithms, wireless sensor network, Mobile Ad hoc Network, Cognitive Radio.

Jayashree Agarkhed, Currently working as professor in the Computer Science and Engineering Department received the B.E. degree from Gulbarga University, M. Tech and Ph. D. degree from Visveshwarai Technological University Karnataka, India, in 1999, 2003 and 2013 respectively. Her research interest is in the area of wireless networking with a QoS provisioning, scheduling and routing algorithm design in sensor network, Ad Hoc Networks and cloud computing.

How to cite this paper: Sujata V. Mallapur, Siddarama R. Patil, Jayashree V. Agarkhed,"A Stable Backbone-Based on Demand Multipath Routing Protocol for Wireless Mobile Ad Hoc Networks", International Journal of Computer Network and Information Security(IJCNIS), Vol.8, No.3, pp.41-51, 2016.DOI: 10.5815/ijcnis.2016.03.06 